\title{
Factors Affecting Patient Satisfaction Related to Cost and Treatment Effectiveness in Rheumatoid Arthritis: Results From the Multicenter Observational Cohort Study, FRANK Registry
}

Toshifumi Fujiwara ( $\nabla$ fujiwara.toshifumi.771@m.kyushu-u.ac.jp )

Kyushu University https://orcid.org/0000-0001-9381-9696

Masakazu Kondo

Kondo Clinic

Hisakata Yamada

Kyushu University

\section{Akihisa Haraguchi}

National Hospital Organisation Kyushu Medical Center: Kokuritsu Byoin Kiko Kyushu Iryo Center

Kenjiro Fujimura

National Hospital Organisation Kyushu Medical Center: Kokuritsu Byoin Kiko Kyushu Iryo Center

Koji Sakuraba

National Hospital Organisation Kyushu Medical Center: Kokuritsu Byoin Kiko Kyushu Iryo Center

\section{Satoshi Kamura}

National Hospital Organisation Kyushu Medical Center: Kokuritsu Byoin Kiko Kyushu Iryo Center

Jun-ichi Fukushi

National Hospital Organisation Kyushu Medical Center: Kokuritsu Byoin Kiko Kyushu Iryo Center

Hisaaki Miyahara

National Hospital Organisation Kyushu Medical Center: Kokuritsu Byoin Kiko Kyushu Iryo Center

Yasushi Inoue

Fukuoka red cross Hospital

Tomomi Tsuru

PS clinic

Toshihide Shuto

Chiyoda Hospital

\section{Seiji Yoshizawa}

Hamanomachi Hopital

\section{Eiichi Suematsu}

National Hospital Organisation Kyushu Medical Center: Kokuritsu Byoin Kiko Kyushu Iryo Center

\section{Tomoya Miyamura}

National Hospital Organisation Kyushu Medical Center: Kokuritsu Byoin Kiko Kyushu Iryo Center Masahiro Ayano

Kyushu University: Kyushu Daigaku

Hiroki Mitoma 
Kyushu University: Kyushu Daigaku

\section{Yojiro Arinobu}

Kyushu University: Kyushu Daigaku

\section{Hiroaki Niiro}

Kyushu University: Kyushu Daigaku

\section{Masanobu Ohishi}

Chihaya Hospital

\section{Akie Hirata}

Kyushu University Hospital: Kyushu Daigaku Byoin

\section{Shoji Tokunaga}

Kyushu University Hospital: Kyushu Daigaku Byoin

\section{Atsushi Takada}

Kyushu University Hospital: Kyushu Daigaku Byoin

\section{Daisuke Hara}

Kyushu Daigaku

\section{Hidetoshi Tsushima}

Kyushu University: Kyushu Daigaku

\section{Yukio Akasaki}

Kyushu University: Kyushu Daigaku

\section{Satoshi Ikemura}

Kyushu University: Kyushu Daigaku

\section{Takuya Sueishi}

Kyushu University: Kyushu Daigaku

\section{Masakazu Toya}

Kyushu University: Kyushu Daigaku

\section{Takahide Sakuragi}

Kyushu university

\section{Tomoko Tsutsui}

Kyushu University: Kyushu Daigaku

\section{Kazuhiro Kai}

Kyushu University: Kyushu Daigaku

\section{Shinkichi Arisumi}

Kyushu University: Kyushu Daigaku

\section{Yasuharu Nakashima}

Kyushu University: Kyushu Daigaku

\section{Research article}

Keywords: rheumatoid arthritis, patient satisfaction, quality of life, observational study, remission, low disease activity

Posted Date: October 29th, 2021 
DOI: https://doi.org/10.21203/rs.3.rs-1006053/v1

License: (c) (i) This work is licensed under a Creative Commons Attribution 4.0 International License. Read Full License

Version of Record: A version of this preprint was published at Arthritis Research \& Therapy on February 22nd, 2022. See the published version at https://doi.org/10.1186/s13075-022-02746-5. 


\section{Abstract}

\section{Background.}

To further improve treatment for rheumatoid arthritis (RA), it is necessary to understand each RA patient satisfaction and to identify the factors affecting patient satisfaction. Despite of escalation of medical cost for RA, the affected factors of satisfaction for cost of treatment in RA patients has been little known.

\section{Methods.}

This is a multicenter observational study of RA patients at Fukuoka, Japan from the FRANK registry, and has been analyzed the initial registered data from March 2017 to August 2020. We collected data on demographic characteristics, clinical data, quality of life, which was assessed using the EuroQol 5-dimensional questionnaire (EQ5D), and patient satisfaction. Patient satisfaction was individually assessed by the four categories (i.e., cost, treatment efficacy, activities of daily living [ADL], and global treatment satisfaction). We analyzed the factors affected with each patient satisfaction, such as age, sex, EQ5D, disease duration, disease activity, and treatment.

\section{Results.}

This study included 2,235 RA outpatients (406 males, 1829 females). At the initial registration, "Very satisfied" and "Satisfied" were given for almost half of each satisfaction aspect (cost: 49\%; effecacy: 72\%; ADL: 58\%; global treatment: 66\%) in RA patients. To investigate the affected factors for each satisfaction, multivariate analysis has revealed that the use of b/tsDMARDs increased satisfaction of treatment effect (odds ratio [OR] 0.67) and ADL (OR 0.79 ), but decreased cost satisfaction (OR 2.24). Aging (50-64 years: OR 0.90, 65-74 years: $0.56, \geq 75$ years: 0.36 ), female (OR 0.80), and history of musculoskeletal surgery (OR 0.60) increased cost satisfaction. All aspects of patient satisfaction were elevated in those with lower disease activity and higher score of EQ5D.

\section{Conclusions.}

Patient satisfaction in terms of cost, treatment effect, ADL, and global treatment was generally better in this study, but still remained some patient with unsatisfaction. Cost of satisfaction was increased with aging and having the history of musculoskeletal surgery, and decreased in lower EQ5D score, and the use of b/tsDMARDs.

\section{Significance And Innovation}

Satisfaction regarding treatment cost was increased in older patients and in those with a history of musculoskeletal surgery.

Lower quality of life and higher disease activity decreased all aspects of patient satisfaction.

The use of bDMARDs increased satisfaction regarding treatment efficacy and activities of daily living, but decreased satisfaction for cost of treatment.

\section{Introduction}

Rheumatoid arthritis (RA) is a progressive inflammatory autoimmune disease that primarily affects the joints and potentially impairs patient's quality of life (QOL). In the last decade, a paradigm shift has been seen in the treatment of RA, the so-called treat-to-target (T2T) strategy, which involves more aggressive, tightly controlled therapy early in 
the disease course guided by a structured assessment of disease activity, with the ultimate goal of reaching remission (1). The advances in understanding the pathogenesis of RA have given rise to new therapeutic target agents. Nowadays, there are several options for the combination of disease-modifying anti-rheumatic drugs (DMARDs), including conventional synthetic DMARDs (csDMARDs), biologic DMARDs (bDMARDs), and targeted synthetic DMARDs (tsDMARDs) (2). These improvements in treatment could dramatically alter patients' QOL and ability to carry out activities of daily living (ADL).

The recent availability of effective therapies has been associated with beneficial outcomes and improved QOL in RA patients, but long-lasting pharmacological treatment may become a chronic burden, especially regarding cost and safety concerns. In the T2T approach for RA treatment, it is necessary that patients comprehend the T2T recommendations and properly communicate with their physicians. However, several reports have presented discordances between patients and physicians regarding their opinions on treatment outcomes, satisfaction, and ADL (3-7). Increased patient satisfaction has influenced RA treatment adherence and continuation $(8,9)$, and this has been enhanced by successful treatments decided on by both the patient and physician $(10,11)$. Thus, understanding patient satisfaction could lead to the overall improvement of RA treatment. Treatment satisfaction of patients with RA has reportedly been improved by the use of bDMARDs, low disease activity, and better communication with their physicians, whereas satisfaction was reduced by unfavorable treatment costs $(12,13)$. High treatment costs have been found to lower patient satisfaction, but it is critical to further characterize patient satisfaction in terms of treatment effectiveness and cost separately in line with the T2T strategy and to eventually improve treatment adherence (14-17). So far, no reports have examined RA patient satisfaction in terms of cost and treatment effectiveness in a large cohort study.

In the present study, we evaluated RA patient satisfaction in terms of cost, treatment effectiveness, current ADL, and global treatment using the initial registration data from a regional observational cohort registry. This study aimed to identify factors influencing satisfaction in RA patients, as well as to investigate factors associated with each dimension of patient satisfaction to describe various aspects of RA treatment.

\section{Materials And Methods}

\section{Data collection}

We established a multicenter prospective observational cohort study of patients with RA at Kyushu University in 2018, using data from the Fukuoka Rheumatoid Arthritis Network (FRANK) registry. The FRANK registry was approved by the ethics committee of Kyushu University (approval number: 29-277). The FRANK registry includes data from a total of nine associated institutions (Kyushu University, Kondo Clinic of Rheumatology and Orthopedic Surgery, National Hospital Organization, Kyushu Medical Center, Fukuoka Red Cross Hospital, PS Clinic, Chiyoda Hospital, Hamanomachi Hospital, and Chihaya Hospital), including clinics and hospitals located in the suburbs of Fukuoka Prefecture in the south of Japan since March 2018. Clinical data were submitted to the data center in Kyushu University Hospital through the Clinical Research Internet Network (CRIN-Q) (18). We enrolled each RA patient during their clinic or hospital visits after obtaining their informed consent. Our analysis included Japanese patients who were at least 18 years old and diagnosed with RA according to the 1987 American College of Rheumatology (ACR) classification criteria. These RA patients have been treated by rheumatologists in accordance with guidelines of the Japan College of Rheumatology. The rheumatologists have annually collected the following demographic and clinical information of each patient: age, sex, body mass index, job, disease duration, Steinbrocker stage, physician visual analog scales (VAS), tender 28-joint count (TJC), swollen 28-joint count (SJC), 
C-reactive protein (CRP) level, erythrocyte sedimentation ratio (ESR), rheumatoid factor positivity, anti-cyclic citrullinated peptide antibody positivity, treatment, and comorbidities. Regarding the present and past treatments, we analyzed the patients' medication use and history of musculoskeletal surgery, such as the use and dose of csDMARDs (e.g., methotrexate), the use and dose of prednisolone, the use of b/tsDMARDs, the number of previously administered b/tsDMARDs, the reasons for discontinuation or non-use of b/tsDMARDs, and the history of musculoskeletal surgery associated with RA, such as arthroplasty $(19,20)$. The enrolled patients were asked to complete the self-administered questionnaires each year. The questionnaires consisted of pain VAS and general VAS, modified health assessment questionnaire (mHAQ) (21), EuroQol 5-dimensional questionnaire (EQ5D) (22), and patient satisfaction questionnaires. The utility index scores of the EQ5D were calculated using the Japanese version of the EQ5D (23).

\section{Patient satisfaction questionnaire contents}

Patient satisfaction was individually assessed in terms of the following four categories: 1) cost of treatment, 2) effect of treatment, 3) ADL, and 4) global treatment. Each self-administered satisfaction questionnaire was assessed using a 6-step score as follows: very satisfied (1 point), satisfied (2 points), somewhat satisfied ( 3 points), somewhat unsatisfied ( 4 points), unsatisfied ( 5 points), and very unsatisfied ( 6 points).

\section{Patients included in the analysis}

A total of 2370 patients were registered from March 2018 to August 2020 in the FRANK registry. All patients were aged 18 years or older at the initial registration. The following patients were excluded: 1) participants who did not fulfill the 2010 ACR/European League Against Rheumatism and the 1987 ACR criteria for RA (N=38), 2) those who lacked data on the four-dimension satisfaction questionnaires $(\mathrm{N}=91), 3)$ those with malignancy upon initial registration $(\mathrm{N}=5)$, and 4) patients with errors in their obtained information ( $\mathrm{N}=1)$. After exclusion, the data of 2235 patients were analyzed in this study (Figure 2). To examine the test-retest reliability of each satisfaction survey, 68 out of 2235 patients were randomly selected and were required to score each aspect of satisfaction during two consecutive visits within 3 months.

\section{Statistical analysis}

The characteristics of the patients included in the analysis were summarized using descriptive statistics. An ordered logistic regression model was used to analyze the association between satisfaction score and the variables that could potentially affect it as follows: age (i.e., $<50,50-64,65-74$, and $\geq 75$ years), sex, RA disease duration (dichotomized at median value), subjective variables (i.e., pain VAS [cm] dichotomized at median value, general VAS [cm] dichotomized at a median value, and EQ5D score categorized in quartiles), and objective variables (i.e., TJC [0 or $\geq 1$ ], SJC [0 or $\geq 1$ ], log-transformed CRP [mg/dL] as a continuous variable, physician VAS [cm] dichotomized at a median value, history of musculoskeletal surgery, dose of prednisolone [over $5 \mathrm{mg}$ or not], and use of b/tsDMARDs). First, we confirmed the association between these patient satisfaction and subjective variables. Afterward, we analyzed the association between these aspects of satisfaction and objective variables. In the multivariate ordered logistic regression analysis (24), two separate models (subjective and objective) were used to assess factors associated with each aspect of satisfaction as explanatory variables; all models included age, sex, and disease duration as common covariates. The odds ratio (OR) was set to increase according to dissatisfaction. We then examined the statistical significance of the interactions between two of the dichotomous explanatory variables, adding product terms of all pairs of the two covariates into the multivariate model. To evaluate the statistical significance of the interaction between the EQ5D score (four indicator variables) and one of the dichotomous 
covariates, a likelihood ratio test was used, comparing a multivariate model that included the four product terms to a model without them. Test-retest reliability was assessed using Kappa statistics. All statistical analyses were performed using the SAS version 9.4 (SAS Institute, Inc., Cary, NC, USA).

\section{Results}

\section{Patient characteristics}

In this study, a total of 2,235 Japanese patients with RA were evaluated from the FRANK registry (Figure 1). Their baseline characteristics and clinical information are shown in Table 1. The median age and disease duration at the initial registration were 65 (range: 19-98) years and 11 (range: 0-70) years, respectively. The each median TJC, SJC, serum CRP, and ESR were 0 (range: 0-28), 0 (range: 0-13), 0.11 (range: 0-21.9) mg/dL, and 14 (range: 0-10) $\mathrm{mm} /$ hour, indicating that almost all patients had lower disease activity in this cohort. The number of patients treated with prednisolone was 896 patients (40\%), methotrexate was used in 1614 patients (72\%), and the use of b/tsDMARDs was seen in 697 patients (31\%). The median mHAQ was 1.1 (range: 1-4), whereas the median EQ5D was 0.8 (range: $0.03-1.0$ ). The distributions for each aspect of satisfaction are shown in Figure 2. Scores of "Very satisfied" (1 point) and "Satisfied" (2 points) were given for almost half of each satisfaction aspect (cost of treatment: 49\%; effect of treatment: $72 \%$; ADL: 58\%; global treatment: $66 \%$ ). The estimates of internal reliability of patient satisfaction analyzed using Kappa statistics were 0.75 for cost, 0.53 for treatment, 0.60 for ADL, and 0.58 for global treatment. 
Table 1

Baseline characteristics of the included patients

\begin{tabular}{|c|c|}
\hline Variables & $N=2235$ \\
\hline Age (years) (median, range) & $65,19-98$ \\
\hline$<50(\%)$ & $381(17 \%)$ \\
\hline $50-65(\%)$ & $699(31 \%)$ \\
\hline $65-74(\%)$ & $767(34 \%)$ \\
\hline$\geq 75(\%)$ & $388(17 \%)$ \\
\hline Sex (male, female) (\%) & $406(18 \%), 1829(82 \%)$ \\
\hline Body mass index (median, range) & $22,13-42$ \\
\hline Job (yes, no) (\%) & $957(45 \%), 1179(55 \%)$ \\
\hline Disease duration (years) (median, range) & $11,0-70$ \\
\hline Steinbrocker Stage 1, 2, 3, 4 (\%) & $612(27 \%), 709$ (32\%), 305 (14\%), 560 (25\%) \\
\hline TJC (median, range) & $0,0-28$ \\
\hline SJC (median, range) & $0,0-13$ \\
\hline RF (positive, negative) & 1574 (73.7\%), $563(26.4 \%)$ \\
\hline ACPA (positive, negative) & 1541 (78.9\%), $411(21.1 \%)$ \\
\hline CRP (mg/dL) (median, range) & $0.11,0-21.9$ \\
\hline ESR 1 hour (mm) (median, range) & $14,0-144$ \\
\hline \multicolumn{2}{|l|}{ Treatment } \\
\hline \multicolumn{2}{|l|}{ Medication } \\
\hline Prednisolone use (yes, no) (\%) & $896(40 \%), 1339(60 \%)$ \\
\hline dose (mg) (median, range) & $3,0-18$ \\
\hline Methotrexate use (yes, no) (\%) & $1614(72 \%), 621(28 \%)$ \\
\hline dose (mg) (median, range) & $8,2-20$ \\
\hline b/tsDMARDs use (yes, no) (\%) & $697(31 \%), 1538(69 \%)$ \\
\hline History of musculoskeletal surgery (yes, no) (\%) & $463(21 \%), 1769(79 \%)$ \\
\hline pain VAS (cm) (median, range) & $1.2,0-10$ \\
\hline general VAS (cm) (median, range) & $0.9,0-10$ \\
\hline physician VAS (cm) (median, range) & $0.5,0-10$ \\
\hline
\end{tabular}

TJC, tender 28-joint count; SJC, swollen 28-joint count; RF, Rheumatoid factor; ACPA, Anti-citrullinated protein/peptide antibody; CRP, C-reactive protein; ESR, erythrocyte sedimentation ratio; b/tsDMARDs, biological/targeted synthetic disease-modifying antirheumatic drugs; VAS, visual analog scales; $\mathrm{mHAQ}$, modified health assessment questionnaire; EQ5D, EuroQol 5 dimensions. 


\begin{tabular}{|lc|}
\hline Variables & $\mathbf{N}=\mathbf{2 2 3 5}$ \\
\hline mHAQ (median, range) & $0.1,0-3.0$ \\
\hline EQ5D score (median, range) & $0.8,0.03-1.0$ \\
\hline $\begin{array}{l}\text { TJC, tender 28-joint count; SJC, swollen 28-joint count; RF, Rheumatoid factor; ACPA, Anti-citrullinated } \\
\text { protein/peptide antibody; CR, C-reactive protein; ESR, erythrocyte sedimentation ratio; b/tsDMARDs, } \\
\text { biological/targeted synthetic disease-modifying antirheumatic drug; VAS, visual analog scales; mHAQ, } \\
\text { modified health assessment questionnaire; EQ5D, EuroQol 5 dimensions. }\end{array}$ \\
\hline
\end{tabular}

\section{Factors associated with satisfaction with cost of treatment}

The univariate and multivariate analyses of the subjective and objective factors associated with satisfaction of cost of treatment are shown in Table 2. Compared with the patients under 50 years old as a reference, satisfaction with the cost of treatment improved with aging in both subjective ( $<65$ years: OR 0.78 [ $p=0.037$ ]; $65-74$ years: OR 0.43 [ $<0.001]$; $\geq 75$ years: OR 0.25 [ $<<0.001]$ ) and objective ( $65-74$ years: OR 0.56 [p < 0.001]; $\geq 75$ years: OR 0.36 [ $<0.001]$ ) factors. Having lower EQ5D score significantly increased dissatisfaction with the cost of treatment (0.9-1.0: referent; 0.85-0.89: OR 1.52 [p = 0.004]; 0.75-0.84: OR 1.54 [p < 0.001]; 0-0.74: OR 1.27 [p = 0.044]). In the multivariate analysis of objective factors, the presence of SJC (OR 1.37 [p = 0.002]), higher physician VAS $(>0.5 ;$ OR $1.34[p=0.003])$, and the use of $b / t s D M A R D s(O R 2.24[p<0.001])$ each significantly elevated dissatisfaction. Interestingly, higher CRP level (OR $0.92[p=0.006])$ and having a history of musculoskeletal surgery (OR $0.60[p<$ 0.001]) significantly increased satisfaction. Significant interactions in the subjective model were observed in disease duration, pain VAS, and EQ5D. Additionally, significant interactions in the objective model were seen for age, sex, disease duration, physician VAS, CRP, history of musculoskeletal surgery, and use of b/tsDMARDs (Table 6). 
Table 2

Factors associated with satisfaction with treatment cost

Univariate

\begin{tabular}{|c|c|c|c|c|c|c|c|c|c|c|}
\hline & & \multirow[b]{2}{*}{ OR } & \multirow[b]{2}{*}{$95 \% \mathrm{Cl}$} & \multirow[b]{2}{*}{$\begin{array}{l}\mathrm{p}- \\
\text { value }\end{array}$} & \multicolumn{3}{|c|}{ Subjective model } & \multicolumn{3}{|c|}{ Objective model } \\
\hline & & & & & OR & $\begin{array}{l}95 \% \\
\mathrm{Cl}\end{array}$ & $\begin{array}{l}\mathrm{p}- \\
\text { value }\end{array}$ & OR & $\begin{array}{l}95 \% \\
\mathrm{Cl}\end{array}$ & $\begin{array}{l}\mathrm{p}- \\
\text { value }\end{array}$ \\
\hline \multicolumn{11}{|l|}{$\begin{array}{l}\text { Common } \\
\text { variables }\end{array}$} \\
\hline \multirow[t]{4}{*}{ Age (years) } & $<50$ & ref & - & - & ref & - & - & ref & - & - \\
\hline & $50-64$ & 0.77 & $\begin{array}{l}0.62- \\
0.97\end{array}$ & 0.023 & 0.78 & $\begin{array}{l}0.62- \\
0.99\end{array}$ & 0.037 & 0.90 & $\begin{array}{l}0.71- \\
1.14\end{array}$ & 0.396 \\
\hline & $65-74$ & 0.45 & $\begin{array}{l}0.36- \\
0.57\end{array}$ & $<0.001$ & 0.43 & $\begin{array}{l}0.34- \\
0.54\end{array}$ & $<0.001$ & 0.56 & $\begin{array}{l}0.44- \\
0.70\end{array}$ & $<0.001$ \\
\hline & $\geq 75$ & 0.27 & $\begin{array}{l}0.21- \\
0.35\end{array}$ & $<0.001$ & 0.25 & $\begin{array}{l}0.19- \\
0.33\end{array}$ & $<0.001$ & 0.36 & $\begin{array}{l}0.27- \\
0.47\end{array}$ & $<0.001$ \\
\hline Sex & Female & 0.97 & $\begin{array}{l}0.80- \\
1.18\end{array}$ & 0.791 & 0.86 & $\begin{array}{l}0.70- \\
1.05\end{array}$ & 0.138 & 0.80 & $\begin{array}{l}0.65- \\
0.99\end{array}$ & 0.038 \\
\hline $\begin{array}{l}\text { Disease duration } \\
\text { (years) }\end{array}$ & $\geq 11$ & 0.89 & $\begin{array}{l}0.77- \\
1.04\end{array}$ & 0.138 & 1.02 & $\begin{array}{l}0.87- \\
1.19\end{array}$ & 0.841 & 1.06 & $\begin{array}{l}0.87- \\
1.28\end{array}$ & 0.584 \\
\hline \multicolumn{11}{|l|}{$\begin{array}{l}\text { Subjective } \\
\text { variables }\end{array}$} \\
\hline pain VAS (cm) & $>1.2$ & 1.34 & $\begin{array}{l}1.15- \\
1.554\end{array}$ & $<0.001$ & 1.19 & $\begin{array}{l}0.98- \\
1.45\end{array}$ & 0.088 & - & - & - \\
\hline $\begin{array}{l}\text { general VAS } \\
(\mathrm{cm})\end{array}$ & $>0.9$ & 1.16 & $\begin{array}{l}1.00- \\
1.35\end{array}$ & 0.047 & 1.01 & $\begin{array}{l}0.84- \\
1.23\end{array}$ & 0.900 & - & - & - \\
\hline \multirow[t]{4}{*}{ EQ5D score } & 0.9-1.0 & ref & - & - & ref & - & - & - & - & - \\
\hline & $\begin{array}{l}0.85- \\
0.89\end{array}$ & 1.51 & $\begin{array}{l}1.14- \\
2.00\end{array}$ & 0.004 & 1.52 & $\begin{array}{l}1.14- \\
2.03\end{array}$ & 0.004 & - & - & - \\
\hline & $\begin{array}{l}0.75- \\
0.84\end{array}$ & 1.49 & $\begin{array}{l}1.22- \\
1.81\end{array}$ & $<0.001$ & 1.54 & $\begin{array}{l}1.24- \\
1.90\end{array}$ & $<0.001$ & - & - & - \\
\hline & $0-0.74$ & 1.17 & $\begin{array}{l}0.97- \\
1.42\end{array}$ & 0.109 & 1.27 & $\begin{array}{l}1.01- \\
1.59\end{array}$ & 0.044 & - & - & - \\
\hline \multicolumn{11}{|l|}{$\begin{array}{l}\text { Objective } \\
\text { variables }\end{array}$} \\
\hline TJC & $\geq 1$ & 1.31 & $\begin{array}{l}1.12- \\
1.53\end{array}$ & $<0.001$ & - & - & - & 1.04 & $\begin{array}{l}0.86- \\
1.27\end{array}$ & 0.674 \\
\hline SJC & $\geq 1$ & 1.42 & $\begin{array}{l}1.28- \\
1.66\end{array}$ & $<0.001$ & - & - & - & 1.37 & $\begin{array}{l}1.13- \\
1.67\end{array}$ & 0.002 \\
\hline CRP (mg/dL) & & 0.88 & $\begin{array}{l}0.84- \\
0.93\end{array}$ & $<0.001$ & - & - & - & 0.92 & $\begin{array}{l}0.87- \\
0.98\end{array}$ & 0.006 \\
\hline
\end{tabular}

\section{Multivariate}




\begin{tabular}{|c|c|c|c|c|c|c|c|c|c|c|}
\hline \multirow[b]{2}{*}{$\begin{array}{l}\text { physician VAS } \\
(\mathrm{cm})\end{array}$} & \multirow[b]{2}{*}{$>0.5$} & \multicolumn{3}{|c|}{ Univariate } & \multicolumn{6}{|c|}{ Multivariate } \\
\hline & & 1.26 & $\begin{array}{l}1.08- \\
1.46\end{array}$ & 0.003 & - & - & - & 1.34 & $\begin{array}{l}1.10- \\
1.61\end{array}$ & 0.003 \\
\hline $\begin{array}{l}\text { Steinbrocker } \\
\text { stage }\end{array}$ & 3 and 4 & 0.87 & $\begin{array}{l}0.74- \\
1.01\end{array}$ & 0.069 & - & - & - & 0.84 & $\begin{array}{l}0.69- \\
1.04\end{array}$ & 0.103 \\
\hline $\begin{array}{l}\text { Musculoskeletal } \\
\text { surgery }\end{array}$ & yes & 0.57 & $\begin{array}{l}0.47- \\
0.69\end{array}$ & $<0.001$ & - & - & - & 0.60 & $\begin{array}{l}0.49- \\
0.74\end{array}$ & $<0.001$ \\
\hline $\begin{array}{l}\text { Prednisolone } \\
\text { dose }(\mathrm{mg})\end{array}$ & $>5$ & 1.14 & $\begin{array}{l}0.81- \\
1.62\end{array}$ & 0.454 & - & - & - & 1.18 & $\begin{array}{l}0.82- \\
1.70\end{array}$ & 0.366 \\
\hline $\begin{array}{l}\mathrm{b} / \mathrm{tsDMARDs} \\
\text { use }\end{array}$ & yes & 2.47 & $\begin{array}{l}2.10- \\
2.91\end{array}$ & $<0.001$ & - & - & - & 2.24 & $\begin{array}{l}1.87- \\
2.68\end{array}$ & $<0.001$ \\
\hline
\end{tabular}

Table 6

Results of instrumental variable analysis for each aspect of satisfaction.

\begin{tabular}{|c|c|c|c|}
\hline Satisfaction & Model & Interacted factors & $\begin{array}{l}\text { p-value for } \\
\text { interaction }\end{array}$ \\
\hline \multirow{7}{*}{$\begin{array}{l}\text { Cost of } \\
\text { Treatment }\end{array}$} & \multirow{2}{*}{$\begin{array}{l}\text { Subjective } \\
\text { model }\end{array}$} & Disease duration and EQ5D & $<0.001$ \\
\hline & & Pain VAS and EQ5D & 0.004 \\
\hline & \multirow{5}{*}{$\begin{array}{l}\text { Objective } \\
\text { model }\end{array}$} & Age and b/tsDMARDs use & 0.013 \\
\hline & & Sex and CRP & $<0.001$ \\
\hline & & $\begin{array}{l}\text { Disease duration and history of musculoskeletal } \\
\text { surgery }\end{array}$ & 0.011 \\
\hline & & CRP and physician VAS & 0.006 \\
\hline & & $\begin{array}{l}\text { History of musculoskeletal surgery and } \\
\text { b/tsDMARDs use }\end{array}$ & $<0.001$ \\
\hline \multirow{3}{*}{$\begin{array}{l}\text { Effect of } \\
\text { Treatment }\end{array}$} & \multirow{2}{*}{$\begin{array}{l}\text { Subjective } \\
\text { model }\end{array}$} & Disease duration and EQ5D & 0.007 \\
\hline & & General VAS and EQ5D & 0.013 \\
\hline & $\begin{array}{l}\text { Objective } \\
\text { model }\end{array}$ & TJC and history of musculoskeletal surgery & 0.034 \\
\hline \multirow[t]{3}{*}{$A D L$} & \multirow{3}{*}{$\begin{array}{l}\text { Subjective } \\
\text { model }\end{array}$} & Disease duration and sex & 0.017 \\
\hline & & Pain VAS and EQ5D & 0.024 \\
\hline & & General VAS and EQ5D & 0.030 \\
\hline \multirow[t]{3}{*}{$\begin{array}{l}\text { Global } \\
\text { Treatment }\end{array}$} & $\begin{array}{l}\text { Subjective } \\
\text { model }\end{array}$ & General VAS and EQ5D & $<0.001$ \\
\hline & \multirow{2}{*}{$\begin{array}{l}\text { Objective } \\
\text { model }\end{array}$} & Age and Steinbrocker stage & 0.029 \\
\hline & & Age and b/tsDMARDs use & 0.005 \\
\hline
\end{tabular}




\section{Factors associated with satisfaction with effect of treatment}

As shown by Table 3, compared with under 50 year-old, satisfaction with the effect of treatment decreased with aging (50-65 years) (OR $1.41[\mathrm{p}=0.006]$ and OR 1.29 [p = 0.044]), high pain VAS (OR $2.63[\mathrm{p}<0.001]$ ) and general VAS (OR 1.28 [p = 0.020]), lower EQ5D score (0.85-0.89: OR 1.90 [p < 0.001]; 0.75-0.84: OR 2.89 [p < 0.001]; 0-0.74: OR 4.55 [p < 0.001]), presence of TJC (OR 1.74 [p < 0.001]) and SJC (OR 1.42 [p < 0.001]), higher CRP level (OR 1.10 [p = 0.003]), elevated physician VAS (OR 2.22 [p < 0.001]), and Steinbrocker stages 3 and $4($ OR 1.32 [p = 0.011]).

The use of $b /$ tsDMARDs improved satisfaction with the effect of treatment (OR 0.67 [p < 0.001$])$. Interactions in the subjective model were significantly seen for disease duration, pain VAS, and EQ5D, while interactions in the objective model were observed for TJC and a history of musculoskeletal surgery (Table 6). 
Table 3

Factors associated with satisfaction with treatment effect

\section{Univariate}

\section{Multivariate}

\begin{tabular}{|c|c|c|c|c|c|c|c|c|c|c|}
\hline & & \multirow[b]{2}{*}{ OR } & \multirow[b]{2}{*}{$\begin{array}{l}95 \% \\
\mathrm{Cl}\end{array}$} & \multirow[b]{2}{*}{$\begin{array}{l}\mathrm{p}- \\
\text { value }\end{array}$} & \multicolumn{3}{|c|}{ Subjective model } & \multicolumn{3}{|c|}{ Objective model } \\
\hline & & & & & OR & $\begin{array}{l}95 \% \\
\mathrm{Cl}\end{array}$ & $\begin{array}{l}\mathrm{p}- \\
\text { value }\end{array}$ & OR & $\begin{array}{l}95 \% \\
\mathrm{Cl}\end{array}$ & $\begin{array}{l}\mathrm{p}- \\
\text { value }\end{array}$ \\
\hline \multicolumn{11}{|l|}{$\begin{array}{l}\text { Common } \\
\text { variables }\end{array}$} \\
\hline \multirow[t]{4}{*}{ Age (years) } & $<50$ & ref & - & - & ref & - & - & - & - & - \\
\hline & $50-64$ & 1.55 & $\begin{array}{l}1.23- \\
1.96\end{array}$ & $<0.001$ & 1.41 & $\begin{array}{l}1.10- \\
1.79\end{array}$ & 0.006 & 1.29 & $\begin{array}{l}1.01- \\
1.65\end{array}$ & 0.044 \\
\hline & $65-74$ & 1.23 & $\begin{array}{l}0.74- \\
1.54\end{array}$ & 0.082 & 1.07 & $\begin{array}{l}0.84- \\
1.36\end{array}$ & 0.606 & 0.96 & $\begin{array}{l}0.75- \\
1.23\end{array}$ & 0.754 \\
\hline & $\geq 75$ & 1.26 & $\begin{array}{l}0.97- \\
1.64\end{array}$ & 0.083 & 0.89 & $\begin{array}{l}0.67- \\
1.18\end{array}$ & 0.409 & 0.86 & $\begin{array}{l}0.65- \\
1.14\end{array}$ & 0.299 \\
\hline Sex & Female & 1.08 & $\begin{array}{l}0.88- \\
1.32\end{array}$ & 0.454 & 0.89 & $\begin{array}{l}0.72- \\
1.10\end{array}$ & 0.288 & 1.06 & $\begin{array}{l}0.86- \\
1.32\end{array}$ & 0.572 \\
\hline $\begin{array}{l}\text { Disease duration } \\
\text { (years) }\end{array}$ & $\geq 11$ & 1.17 & $\begin{array}{l}1.00- \\
1.32\end{array}$ & 0.050 & 0.94 & $\begin{array}{l}0.80- \\
1.11\end{array}$ & 0.481 & 0.96 & $\begin{array}{l}0.79- \\
1.17\end{array}$ & 0.668 \\
\hline \multicolumn{11}{|l|}{$\begin{array}{l}\text { Subjective } \\
\text { variables }\end{array}$} \\
\hline pain VAS (cm) & $>1.2$ & 5.00 & $\begin{array}{l}4.20- \\
5.94\end{array}$ & $<0.001$ & 2.63 & $\begin{array}{l}2.12- \\
3.26\end{array}$ & $<0.001$ & - & - & - \\
\hline general VAS (cm) & $>0.9$ & 3.31 & $\begin{array}{l}2.81- \\
3.91\end{array}$ & $<0.001$ & 1.28 & $\begin{array}{l}1.04- \\
1.57\end{array}$ & 0.020 & - & - & - \\
\hline \multirow[t]{4}{*}{ EQ5D score } & $0.9-1.0$ & ref & - & - & ref & - & - & - & - & - \\
\hline & $\begin{array}{l}0.85- \\
0.89\end{array}$ & 2.37 & $\begin{array}{l}1.76- \\
3.19\end{array}$ & $<0.001$ & 1.90 & $\begin{array}{l}1.40- \\
2.59\end{array}$ & $<0.001$ & - & - & - \\
\hline & $\begin{array}{l}0.75- \\
0.84\end{array}$ & 3.90 & $\begin{array}{l}3.14- \\
4.84\end{array}$ & $<0.001$ & 2.89 & $\begin{array}{l}2.29- \\
3.64\end{array}$ & $<0.001$ & - & - & - \\
\hline & $0-0.74$ & 7.72 & $\begin{array}{l}6.21- \\
9.59\end{array}$ & $<0.001$ & 4.55 & $\begin{array}{l}3.54- \\
5.84\end{array}$ & $<0.001$ & - & - & - \\
\hline \multicolumn{11}{|l|}{$\begin{array}{l}\text { Objective } \\
\text { variables }\end{array}$} \\
\hline TJC & $\geq 1$ & 3.13 & $\begin{array}{l}2.64- \\
3.71\end{array}$ & $<0.001$ & - & - & - & 1.74 & $\begin{array}{l}1.43- \\
2.13\end{array}$ & $<0.001$ \\
\hline SJC & $\geq 1$ & 2.82 & $\begin{array}{l}2.38- \\
3.34\end{array}$ & $<0.001$ & - & - & - & 1.42 & $\begin{array}{l}1.16- \\
1.74\end{array}$ & $<0.001$ \\
\hline CRP (mg/dL) & & 1.29 & $\begin{array}{l}1.22- \\
1.36\end{array}$ & $<0.001$ & - & - & - & 1.10 & $\begin{array}{l}1.03- \\
1.17\end{array}$ & 0.003 \\
\hline $\begin{array}{l}\text { physician VAS } \\
(\mathrm{cm})\end{array}$ & $>0.5$ & 3.62 & $\begin{array}{l}3.06- \\
4.27\end{array}$ & $<0.001$ & - & - & - & 2.22 & $\begin{array}{l}1.81- \\
2.71\end{array}$ & $<0.001$ \\
\hline
\end{tabular}




\begin{tabular}{|c|c|c|c|c|c|c|c|c|c|c|}
\hline \multirow[b]{2}{*}{$\begin{array}{l}\text { Steinbrocker } \\
\text { stage }\end{array}$} & \multirow[b]{2}{*}{3 and 4} & \multicolumn{3}{|c|}{ Univariate } & \multicolumn{6}{|c|}{ Multivariate } \\
\hline & & 1.59 & $\begin{array}{l}1.36- \\
1.87\end{array}$ & $<0.001$ & - & - & - & 1.32 & $\begin{array}{l}1.07- \\
1.63\end{array}$ & 0.011 \\
\hline $\begin{array}{l}\text { Musculoskeletal } \\
\text { surgery }\end{array}$ & yes & 1.58 & $\begin{array}{l}1.30- \\
1.91\end{array}$ & $<0.001$ & - & - & - & 1.10 & $\begin{array}{l}0.89- \\
1.37\end{array}$ & 0.373 \\
\hline $\begin{array}{l}\text { Prednisolone } \\
\text { dose }(\mathrm{mg})\end{array}$ & $>5$ & 1.99 & $\begin{array}{l}1.39- \\
2.84\end{array}$ & $<0.001$ & - & - & - & 1.29 & $\begin{array}{l}0.89- \\
1.88\end{array}$ & 0.181 \\
\hline $\mathrm{b} / \mathrm{tsDMARDs}$ use & yes & 0.75 & $\begin{array}{l}0.63- \\
0.89\end{array}$ & $<0.001$ & - & - & - & 0.67 & $\begin{array}{l}0.56- \\
0.81\end{array}$ & $<0.001$ \\
\hline
\end{tabular}

\section{Factors associated with satisfaction with ADL}

Compared with under 50 year-old, dissatisfaction with ADL increased with aging (50-64 years) (OR 1.55 [p < 0.001$]$ and OR 1.52 [p < 0.001]), longer disease duration ( $\geq 11$; OR $1.25[p=0.009])$, high pain VAS (OR $2.63[p<0.001])$ and general VAS (OR 1.35 [p = 0.003]), lower EQ5D score (0.85-<0.9: OR 3.24 [p < 0.001]; 0.75-<0.85: OR 3.90 [p< 0.001]; <0.75: OR $9.96[p<0.001])$, presence of TJC (OR 2.02 [p < 0.001]) and SJC (OR 1.34 [p = 0.003]), higher CRP level (OR 1.09 [p = 0.004]), elevated VAS physician (OR 2.42 [p < 0.001]), Steinbrocker stages 3 and 4 (OR 1.56 [p < $0.001])$, history of musculoskeletal surgery (OR $1.40[p=0.002])$, and higher dose of prednisolone (OR $1.54[p=$ 0.022]) (Table 4). On the other hand, the use of b/tsDMARDs increased satisfaction with ADL of RA patients (OR $0.79[p=0.011])$. Sex, disease duration, VAS pain, VAS general, and EQ5D were statistically interacted in the subjective model (Table 6). 
Table 4

Factors associated with satisfaction with activities of daily living

\section{Univariate}

Multivariate

\begin{tabular}{|c|c|c|c|c|c|c|c|c|c|c|}
\hline & & \multirow[b]{2}{*}{ OR } & \multirow[b]{2}{*}{$95 \% \mathrm{Cl}$} & \multirow[b]{2}{*}{$\begin{array}{l}\mathrm{p}- \\
\text { value }\end{array}$} & \multicolumn{3}{|c|}{ Subjective model } & \multicolumn{3}{|c|}{ Objective model } \\
\hline & & & & & OR & $\begin{array}{l}95 \% \\
\mathrm{Cl}\end{array}$ & $\begin{array}{l}\mathrm{p}- \\
\text { value }\end{array}$ & OR & $\begin{array}{l}95 \% \\
\mathrm{Cl}\end{array}$ & $\begin{array}{l}\mathrm{p}- \\
\text { value }\end{array}$ \\
\hline \multicolumn{11}{|l|}{$\begin{array}{l}\text { Common } \\
\text { variables }\end{array}$} \\
\hline \multirow[t]{4}{*}{ Age (years) } & $<50$ & ref & - & - & ref & - & - & ref & - & - \\
\hline & $50-64$ & 1.67 & $\begin{array}{l}1.33- \\
2.10\end{array}$ & $<0.001$ & 1.55 & $\begin{array}{l}1.22- \\
1.98\end{array}$ & $<0.001$ & 1.52 & $\begin{array}{l}1.19- \\
1.93\end{array}$ & $<0.001$ \\
\hline & $65-74$ & 1.40 & $\begin{array}{l}1.12- \\
1.76\end{array}$ & 0.003 & 1.23 & $\begin{array}{l}0.97- \\
1.57\end{array}$ & 0.089 & 1.16 & $\begin{array}{l}0.91- \\
1.48\end{array}$ & 0.239 \\
\hline & $\geq 75$ & 1.56 & $\begin{array}{l}1.21- \\
2.02\end{array}$ & $<0.001$ & 1.01 & $\begin{array}{l}0.76- \\
1.34\end{array}$ & 0.948 & 1.15 & $\begin{array}{l}0.87- \\
1.55\end{array}$ & 0.324 \\
\hline Sex & Female & 1.33 & $\begin{array}{l}1.09- \\
1.61\end{array}$ & 0.005 & 1.03 & $\begin{array}{l}0.83- \\
1.27\end{array}$ & 0.792 & 1.19 & $\begin{array}{l}0.96- \\
1.47\end{array}$ & 0.110 \\
\hline $\begin{array}{l}\text { Disease } \\
\text { duration (years) }\end{array}$ & $\geq 11$ & 1.59 & $\begin{array}{l}1.36- \\
1.85\end{array}$ & $<0.001$ & 1.25 & $\begin{array}{l}1.06- \\
1.47\end{array}$ & 0.009 & 1.14 & $\begin{array}{l}0.94- \\
1.39\end{array}$ & 0.188 \\
\hline \multicolumn{11}{|l|}{$\begin{array}{l}\text { Subjective } \\
\text { variables }\end{array}$} \\
\hline pain VAS (cm) & $>1.2$ & 6.15 & $\begin{array}{l}5.18- \\
7.30\end{array}$ & $<0.001$ & 2.63 & $\begin{array}{l}2.13- \\
3.24\end{array}$ & $<0.001$ & - & - & - \\
\hline $\begin{array}{l}\text { general VAS } \\
(\mathrm{cm})\end{array}$ & $>0.9$ & 4.19 & $\begin{array}{l}3.56- \\
4.93\end{array}$ & $<0.001$ & 1.35 & $\begin{array}{l}1.12- \\
1.66\end{array}$ & 0.003 & - & - & - \\
\hline \multirow[t]{4}{*}{ EQ5D socre } & 0.9-1.0 & ref & - & - & ref & - & - & - & - & - \\
\hline & $\begin{array}{l}0.85- \\
0.89\end{array}$ & 3.96 & $\begin{array}{l}2.94- \\
5.33\end{array}$ & $<0.001$ & 3.24 & $\begin{array}{l}2.38- \\
4.41\end{array}$ & $<0.001$ & - & - & - \\
\hline & $\begin{array}{l}0.75- \\
0.84\end{array}$ & 5.49 & $\begin{array}{l}4.41- \\
6.83\end{array}$ & $<0.001$ & 3.90 & $\begin{array}{l}3.09- \\
4.92\end{array}$ & $<0.001$ & - & - & - \\
\hline & $0-0.74$ & 18.11 & $\begin{array}{l}14.37- \\
22.81\end{array}$ & $<0.001$ & 9.96 & $\begin{array}{l}7.7- \\
12.87\end{array}$ & $<0.001$ & - & - & - \\
\hline \multicolumn{11}{|l|}{$\begin{array}{l}\text { Objective } \\
\text { variables }\end{array}$} \\
\hline TJC & $\geq 1$ & 3.70 & $\begin{array}{l}3.13- \\
4.37\end{array}$ & $<0.001$ & - & - & - & 2.02 & $\begin{array}{l}1.66- \\
2.46\end{array}$ & $<0.001$ \\
\hline SJC & $\geq 1$ & 3.11 & $\begin{array}{l}2.63- \\
3.68\end{array}$ & $<0.001$ & - & - & - & 1.34 & $\begin{array}{l}1.10- \\
1.64\end{array}$ & 0.003 \\
\hline $\mathrm{CRP}(\mathrm{mg} / \mathrm{dL})$ & & 1.29 & $\begin{array}{l}1.22- \\
1.36\end{array}$ & $<0.001$ & - & - & - & 1.09 & $\begin{array}{l}1.03- \\
1.16\end{array}$ & 0.004 \\
\hline $\begin{array}{l}\text { physician VAS } \\
(\mathrm{cm})\end{array}$ & $>0.5$ & 4.30 & $\begin{array}{l}3.65- \\
5.07\end{array}$ & $<0.001$ & - & - & - & 2.42 & $\begin{array}{l}1.99- \\
2.94\end{array}$ & $<0.001$ \\
\hline
\end{tabular}

\begin{tabular}{|c|c|c|c|c|c|c|c|c|c|c|}
\hline Age (years) & $<50$ & ref & - & - & ref & - & - & ref & - & - \\
\hline & $50-64$ & 1.67 & $\begin{array}{l}1.33- \\
2.10\end{array}$ & $<0.001$ & 1.55 & $\begin{array}{l}1.22- \\
1.98\end{array}$ & $<0.001$ & 1.52 & $\begin{array}{l}1.19- \\
1.93\end{array}$ & $<0.001$ \\
\hline & $65-74$ & 1.40 & $\begin{array}{l}1.12- \\
1.76\end{array}$ & 0.003 & 1.23 & $\begin{array}{l}0.97- \\
1.57\end{array}$ & 0.089 & 1.16 & $\begin{array}{l}0.91- \\
1.48\end{array}$ & 0.239 \\
\hline & $\geq 75$ & 1.56 & $\begin{array}{l}1.21- \\
2.02\end{array}$ & $<0.001$ & 1.01 & $\begin{array}{l}0.76- \\
1.34\end{array}$ & 0.948 & 1.15 & $\begin{array}{l}0.87- \\
1.55\end{array}$ & 0.324 \\
\hline Sex & Female & 1.33 & $\begin{array}{l}1.09- \\
1.61\end{array}$ & 0.005 & 1.03 & $\begin{array}{l}0.83- \\
1.27\end{array}$ & 0.792 & 1.19 & $\begin{array}{l}0.96- \\
1.47\end{array}$ & 0.110 \\
\hline $\begin{array}{l}\text { Disease } \\
\text { duration (years) }\end{array}$ & $\geq 11$ & 1.59 & $\begin{array}{l}1.36- \\
1.85\end{array}$ & $<0.001$ & 1.25 & $\begin{array}{l}1.06- \\
1.47\end{array}$ & 0.009 & 1.14 & $\begin{array}{l}0.94- \\
1.39\end{array}$ & 0.188 \\
\hline \multicolumn{11}{|l|}{$\begin{array}{l}\text { Subjective } \\
\text { variables }\end{array}$} \\
\hline pain VAS (cm) & $>1.2$ & 6.15 & $\begin{array}{l}5.18- \\
7.30\end{array}$ & $<0.001$ & 2.63 & $\begin{array}{l}2.13- \\
3.24\end{array}$ & $<0.001$ & - & - & - \\
\hline $\begin{array}{l}\text { general VAS } \\
(\mathrm{cm})\end{array}$ & $>0.9$ & 4.19 & $\begin{array}{l}3.56- \\
4.93\end{array}$ & $<0.001$ & 1.35 & $\begin{array}{l}1.12- \\
1.66\end{array}$ & 0.003 & - & - & - \\
\hline \multirow[t]{4}{*}{ EQ5D socre } & $0.9-1.0$ & ref & - & - & ref & - & - & - & - & - \\
\hline & $\begin{array}{l}0.85- \\
0.89\end{array}$ & 3.96 & $\begin{array}{l}2.94- \\
5.33\end{array}$ & $<0.001$ & 3.24 & $\begin{array}{l}2.38- \\
4.41\end{array}$ & $<0.001$ & - & - & - \\
\hline & $\begin{array}{l}0.75- \\
0.84\end{array}$ & 5.49 & $\begin{array}{l}4.41- \\
6.83\end{array}$ & $<0.001$ & 3.90 & $\begin{array}{l}3.09- \\
4.92\end{array}$ & $<0.001$ & - & - & - \\
\hline & $0-0.74$ & 18.11 & $\begin{array}{l}14.37- \\
22.81\end{array}$ & $<0.001$ & 9.96 & $\begin{array}{l}7.7- \\
12.87\end{array}$ & $<0.001$ & - & - & - \\
\hline \multicolumn{11}{|l|}{$\begin{array}{l}\text { Objective } \\
\text { variables }\end{array}$} \\
\hline TJC & $\geq 1$ & 3.70 & $\begin{array}{l}3.13- \\
4.37\end{array}$ & $<0.001$ & - & - & - & 2.02 & $\begin{array}{l}1.66- \\
2.46\end{array}$ & $<0.001$ \\
\hline SJC & $\geq 1$ & 3.11 & $\begin{array}{l}2.63- \\
3.68\end{array}$ & $<0.001$ & - & - & - & 1.34 & $\begin{array}{l}1.10- \\
1.64\end{array}$ & 0.003 \\
\hline $\mathrm{CRP}(\mathrm{mg} / \mathrm{dL})$ & & 1.29 & $\begin{array}{l}1.22- \\
1.36\end{array}$ & $<0.001$ & - & - & - & 1.09 & $\begin{array}{l}1.03- \\
1.16\end{array}$ & 0.004 \\
\hline $\begin{array}{l}\text { physician VAS } \\
(\mathrm{cm})\end{array}$ & $>0.5$ & 4.30 & $\begin{array}{l}3.65- \\
5.07\end{array}$ & $<0.001$ & - & - & - & 2.42 & $\begin{array}{l}1.99- \\
2.94\end{array}$ & $<0.001$ \\
\hline
\end{tabular}

Page 15/25 


\begin{tabular}{|lllllllllll|}
\hline \multicolumn{9}{c|}{ Univariate } & \multicolumn{7}{c|}{ Multivariate } & & & \\
\hline $\begin{array}{l}\text { Steinbrocker } \\
\text { stage }\end{array}$ & 3 and & 2.21 & $\begin{array}{l}1.89- \\
2.59\end{array}$ & $<0.001$ & - & - & - & 1.56 & $1.27-$ & $<0.001$ \\
\hline $\begin{array}{l}\text { Musculoskeletal } \\
\text { surgery }\end{array}$ & yes & 2.21 & $\begin{array}{l}1.83- \\
2.66\end{array}$ & $<0.001$ & - & - & - & 1.40 & $1.13-$ & 0.002 \\
\hline $\begin{array}{l}\text { Prednisolone } \\
\text { dose (mg) }\end{array}$ & $>5$ & 2.26 & $1.60-$ & $<0.001$ & - & - & - & 1.54 & $1.07-$ & 0.022 \\
$\begin{array}{l}\text { b/tsDMARDs } \\
\text { use }\end{array}$ & yes & 0.98 & $\begin{array}{l}3.21 \\
0.83-\end{array}$ & 0.773 & - & - & - & 0.79 & $0.66-$ & 0.011 \\
\hline
\end{tabular}

\section{Factors associated with for satisfaction with global treatment}

Relative to patients younger than 50 years old, satisfaction with global treatment improved with aging both in subjective (65-74 years: OR 0.71 [ $p=0.007] ; \geq 75$ years: OR 0.55 [ $<0.001])$ and objective $(65-74$ years: OR 0.76 $[p=0.031] ; \geq 75:$ OR $0.67[p=0.006])$ factors (Table 5). Satisfaction with global treatment was worse with high VAS pain (OR 2.16 [p < 0.001]), low EQ5D score (0.85-<0.9: OR 2.14 [p < 0.001]; 0.75-<0.85: OR 2.79 [p < 0.001]; <0.75: OR 5.93 [p < 0.001]), presence of TJC (OR 1.70 [p < 0.001]) and SJC (OR 1.25 [p = 0.033]), higher CRP level (OR 1.09 [p = 0.008]), and high VAS physician (OR 2.26 [p < 0.001]). Interactions in the subjective model were significantly seen for VAS pain and EQ5D, whereas those in the objective model were observed for age, Steinbrocker stage, and use of b/tsDMARDs (Table 6). 
Table 5

Factors associated with global treatment satisfaction

Univariate

\begin{tabular}{|c|c|c|c|c|c|c|c|c|}
\hline & & & Sub & ive $\mathrm{m}$ & & Obje & ve mo & \\
\hline OR & $95 \% \mathrm{Cl}$ & $\begin{array}{l}\mathrm{p}- \\
\text { value }\end{array}$ & OR & $\begin{array}{l}95 \% \\
\mathrm{Cl}\end{array}$ & $\begin{array}{l}\mathrm{p}- \\
\text { value }\end{array}$ & OR & $\begin{array}{l}95 \% \\
\mathrm{Cl}\end{array}$ & \\
\hline
\end{tabular}

\section{Common}

variables

Age (years)

$<50$ ref

\section{Multivariate}

50-64 1.15

$65-74$

0.87

$$
65
$$

$$
\geq 75
$$

$0.87 \quad 0.69-$

1.09

$0.87 \quad 0.66-$

1.13

0.284

0.034

Female

Sex

Disease duration $\quad \geq 11$

(years)

$1.24 \quad 1.02-\quad 0.034$

1.52

$1.28 \quad 1.09-$

1.49

0.002

\section{Subjective}

\begin{tabular}{|c|c|c|c|c|c|c|c|c|}
\hline pain VAS (cm) & $>1.2$ & 4.33 & $\begin{array}{l}3.65- \\
5.14\end{array}$ & $<0.001$ & 2.16 & $\begin{array}{l}1.74- \\
2.67\end{array}$ & $<0.001$ & - \\
\hline general VAS (cm) & $>0.9$ & 3.02 & $\begin{array}{l}2.56- \\
3.55\end{array}$ & $<0.001$ & 1.18 & $\begin{array}{l}0.96- \\
1.45\end{array}$ & 0.115 & - \\
\hline \multirow[t]{4}{*}{ EQ5D score } & $0.9-1.0$ & ref & - & - & ref & - & - & - \\
\hline & $\begin{array}{l}0.85- \\
0.89\end{array}$ & 2.51 & $\begin{array}{l}1.85- \\
3.39\end{array}$ & $<0.001$ & 2.14 & $\begin{array}{l}1.56- \\
2.92\end{array}$ & $<0.001$ & - \\
\hline & $\begin{array}{l}0.75- \\
0.84\end{array}$ & 3.46 & $\begin{array}{l}2.78- \\
4.30\end{array}$ & $<0.001$ & 2.79 & $\begin{array}{l}2.21- \\
3.52\end{array}$ & $<0.001$ & - \\
\hline & $0-0.74$ & 8.60 & $\begin{array}{l}6.90- \\
10.71\end{array}$ & $<0.001$ & 5.93 & $\begin{array}{l}4.60- \\
7.65\end{array}$ & $<0.001$ & - \\
\hline
\end{tabular}

variables

Objective

\begin{tabular}{|c|c|c|c|c|c|c|c|c|c|c|}
\hline TJC & $\geq 1$ & 2.91 & $\begin{array}{l}2.46- \\
3.44\end{array}$ & $<0.001$ & - & - & - & 1.70 & $\begin{array}{l}1.39- \\
2.08\end{array}$ & $<0.001$ \\
\hline SJC & $\geq 1$ & 2.48 & $\begin{array}{l}2.09- \\
2.93\end{array}$ & $<0.001$ & - & - & - & 1.25 & $\begin{array}{l}1.02- \\
1.53\end{array}$ & 0.033 \\
\hline $\mathrm{CRP}(\mathrm{mg} / \mathrm{dL})$ & & 1.23 & $\begin{array}{l}1.16- \\
1.30\end{array}$ & $<0.001$ & - & - & - & 1.09 & $\begin{array}{l}1.02- \\
1.16\end{array}$ & 0.008 \\
\hline $\begin{array}{l}\text { physician VAS } \\
(\mathrm{cm})\end{array}$ & $>0.5$ & 3.39 & $\begin{array}{l}2.87- \\
4.00\end{array}$ & $<0.001$ & - & - & - & 2.26 & $\begin{array}{l}1.85- \\
2.76\end{array}$ & $<0.001$ \\
\hline
\end{tabular}

variables

Page $17 / 25$ 


\begin{tabular}{|c|c|c|c|c|c|c|c|c|c|c|}
\hline \multirow[b]{2}{*}{$\begin{array}{l}\text { Steinbrocker } \\
\text { stage }\end{array}$} & \multirow[b]{2}{*}{3 and 4} & \multicolumn{3}{|c|}{ Univariate } & \multicolumn{6}{|c|}{ Multivariate } \\
\hline & & 1.56 & $\begin{array}{l}1.33- \\
1.83\end{array}$ & $<0.001$ & - & - & - & 1.15 & $\begin{array}{l}0.93- \\
1.42\end{array}$ & 0.195 \\
\hline $\begin{array}{l}\text { Musculoskeletal } \\
\text { surgery }\end{array}$ & yes & 1.51 & $\begin{array}{l}1.25- \\
1.83\end{array}$ & $<0.001$ & - & - & - & 1.09 & $\begin{array}{l}0.88- \\
1.35\end{array}$ & 0.431 \\
\hline $\begin{array}{l}\text { Prednisolone } \\
\text { dose }(\mathrm{mg})\end{array}$ & $>5$ & 1.85 & $\begin{array}{l}1.30- \\
2.65\end{array}$ & $<0.001$ & - & - & - & 1.26 & $\begin{array}{l}0.87- \\
1.84\end{array}$ & 0.220 \\
\hline $\mathrm{b} / \mathrm{tsDMARDs}$ use & yes & 1.07 & $\begin{array}{l}0.91- \\
1.27\end{array}$ & 0.423 & - & - & - & 0.95 & $\begin{array}{l}0.79- \\
1.15\end{array}$ & 0.605 \\
\hline
\end{tabular}

\section{Discussion}

Since there are currently no large cohort studies investigating patient satisfaction focusing on the cost of treatment, we separately evaluated patient satisfaction regarding different aspects (i.e., cost of treatment, effect of treatment, $A D L$, and global treatment) using the data of 2,235 RA patients. We identified several patient-related factors affecting each aspect of satisfaction on multivariate analysis.

Because T2T treatment could achieve low disease activity and remission, the majority of the participants in the FRANK registry indicated that they were "very satisfied" or "satisfied" with their current treatment for cost and effectiveness, $A D L$, and global treatment upon initial registration, yet there were still "unsatisfied" patients (Figure 2). Although a few studies have analyzed RA patient satisfaction using a large cohort, a German biological register study of 10,646 RA patients using b/tsDMARDs demonstrated that tapering glucocorticoids was positively associated with patient satisfaction (10). Furthermore, because treatment costs were negatively related with satisfaction (14-16) and because the health care costs of RA patients are higher than those of controls matched for age, sex, and medical history (25), it is important to understand their satisfaction regarding the cost of long periods of RA treatment. In a Chinese registry of RA, patients with a low proportion of treatment cost to income were clearly more satisfied compared with those with a high proportion (17). Therefore, we examined patient satisfaction with the cost of treatment separately. The factors associated with increased cost satisfaction were aging, high EQ5D score, absence of SJC, elevated serum CRP level, history of musculoskeletal surgery, and nonuse of b/tsDMARDs (Table 2). On the other hand, factors such as aging, higher disease activity (each VAS, presence of TJC or SJC, elevated serum CRP level), Steinbrocker stages 3 and 4, a history of musculoskeletal surgery, and higher prednisolone dose, were associated with lower satisfaction with treatment, ADL, and global treatment.

We found that cost satisfaction increased with aging in both subjective and objective models of the multivariate analysis. In the Japanese health care system, the government usually covers $70 \%$ of treatment costs, whereas patients are responsible for $30 \%$. However, co-payment has been adjusted to $10-30 \%$ depending on the family's income and age (<65 years: $30 \% ; 65-74$ years: $20 \%$; $\geq 75$ years: $10 \%$ co-payment). Thus, since older patients pay a lower percentage of co-payment than younger patients, cost satisfaction may markedly increase with age. In addition, patients with a history of musculoskeletal surgery were more satisfied with cost of treatment. Patients with disabilities are given medical coverage by the government, suggesting that patients with a history of musculoskeletal surgery might have increased satisfaction with cost due to the presence of some disabilities. QOL of RA patients was evaluated by EQ5D score (26). In agreement with previous evidence $(7,13,27,28)$, EQ5D score in our cohort also affected all aspects of satisfaction (treatment cost, efficacy, ADL, and global treatment), indicating that the improvement of EQ5D should be critical for better RA treatment and patient satisfaction. 
Because EQ5D showed interactions with disease duration, VAS pain, and VAS general in terms of treatment cost, treatment effect, $A D L$, and global treatment, the satisfaction associated with EQ5D can be separately assessed by each factor. Treatment with b/tsDMARDs dramatically improves treatment outcomes and QOL in RA patients (2931), and the T2T strategy using b/tsDMARDs has been recommended. Better QOL and b/tsDMARDs have been established as positive predictors of global treatment satisfaction. Indeed, the use of b/tsDMARDs increased satisfaction regarding treatment effect and ADL in our cohort, but decreased cost satisfaction significantly. Because the elevated cost of b/tsDMARDs is a burden on health care budgets, the use of biosimilars has been widely approved with reported benefits, such as cost-effectiveness (32-37). However, only few biosimilars were included in our cohort. Therefore, the increased use of biosimilars might affect cost satisfaction in our cohort. Because the use of $b /$ tsDMARDs interacted with age and history of musculoskeletal surgery in terms of satisfaction with cost and global treatment (Table 6), these aspects of satisfaction might be affected by these factors.

As several reports have shown, disease activity influences treatment effectiveness and patient satisfaction, while treatment cost is an independent factor of treatment satisfaction $(10,12,13,38)$. Specifically, high disease activity decreases treatment satisfaction. In agreement with these previous data, in our cohort, disease activity, including SJC, TJC, each VAS, and serum CRP level correlated with increased dissatisfaction regarding treatment effect, ADL, and global treatment. On the other hand, only elevated serum CRP level decreased dissatisfaction with cost of treatment. Although we could not determine the reason, the median serum CRP level $(0.11 \mathrm{mg} / \mathrm{dL})$ in our cohort was low, indicating that slightly elevated serum CRP level might provide good cost performance for RA patients.

Schäfer M. et al. (10) demonstrated that tapering prednisolone was positively associated with satisfaction in RA patients using b/tsDMARDs. Treatment with prednisolone, especially low doses of prednisolone, is safe and effective (39), but continuous use of higher doses should be avoided due to risk of infection, osteoporosis, diabetes mellitus, thrombotic stroke, cardiovascular diseases, and death (40-43). However, discontinuation of prednisolone has been achieved in only nearly half of 508 patients with early RA in recent study (39); the inability to taper prednisolone has been reported as one of the characteristics of difficult-to-treat RA (44). In this study, less than half of the patients with RA (40\%) were treated with prednisolone to control disease activity, suggesting that these patients might have difficult-to-treat RA. This may imply a decreased capacity to perform ADL, indicating that the use of higher doses of prednisolone led to decreased ADL satisfaction.

Interestingly, aging revealed differences in the aspects of satisfaction. With age, we found higher treatment cost and global treatment satisfaction, but lower treatment effect and ADL satisfaction. Previous studies have shown lower EQ5D scores with aging due to poorer physical function and capacity to perform $\operatorname{ADL}(45,46)$, leading to decreased life satisfaction. In our study, satisfaction regarding treatment effect and ADL was diminished in the age group of 50-64 years old in particular. The lower cost of treatment and disease activity in our cohort might have increased global treatment satisfaction with aging. Previous evidence has shown that longer disease duration of RA was associated with impaired ADL (47). Similarly, longer disease duration and higher Steinbrocker stage were associated with lower ADL satisfaction in our study.

This study has limitations. First, because this was a cross-sectional study using data from the initial registrants of the FRANK registry, the changes in satisfaction were unclear. We will elucidate these changes hereafter. Second, our cohort mostly included patients with low disease activity and remission. The T2T strategy has widely spread due to the use of csDMARDs and bDMARDs, resulting in more patients achieving lower disease activity. However, there are still patients with dissatisfaction regarding the effect of treatment, cost of treatment, ADL, and global treatment, 
and thus the analysis of satisfaction in RA patients is important and meaningful in order to further improve their treatment.

\section{Conclusion}

We have conducted a large cohort observational study using patients in the FRANK registry in the south of Japan. We assessed patient satisfaction in terms of cost, treatment effect, ADL, and global treatment in Japanese RA patients, and identified factors influencing each of these aspects. Aging was associated with increased cost and global treatment satisfaction, but also with decreased treatment and ADL satisfaction. Female sex and having a history of musculoskeletal surgery improved cost satisfaction, whereas having a lower EQ5D score and higher disease activity were associated with lower satisfaction in all aspects. The use of b/tsDMARDs decreased cost satisfaction, but increased treatment effect and ADL satisfaction.

\section{Abbreviations}

DMARD: disease-modifying anti-rheumatic drug; RA: rheumatoid arthritis; ADL: activity of daily living; EQ5D:

EuroQol 5-dimensional questionnaire tumor; QOL: quality of life; T2T: treat-to-target; FRANK: Fukuoka rheumatoid arthritis network; VAS: visual analog scales; TJC: tender 28-joint count; SJC: swollen 28-joint count, CRP: C-reactive protein; ESR: erythrocyte sedimentation ratio (ESR), mHAQ: modified health assessment questionnaire; OR: odds ratio

\section{Declarations}

Acknowledgments: The authors would like to thank Enago (www.enago.jp) for the English language review.

Author Contributions: Conceptualization, TF, MK, HY, AH, KF, KS, SK, JF, HM, YI, TT, TS, SY, TM, MA, HM, YA, HN, MO, HT, YA, IS, TS, DH, MT, TS, TT, KK, SA, and YN; Acquisition of data, TF, MK, HY, AH, KF, KS, SK, JF, HM, YI, TT, TS, SY, TM, MA, HM, MA, YA, HN, MO, HT, YA, IS, TS, MT, TS, TT, KK, SA, and YN; Analysis of data, TF, AH, ST, and AT; Interpretation of data, TF, AH, ST, HT, YA, IS, and YN; Original draft preparation, TF, AH, ST, HT, YA, IS, and YN; Writing-reviewing and editing, TF, MK, HY, AH, KF, SK, JF, HM, YI, TT, TS, SY, TM, MA, HM, YA, HN, MO, HT, YA, IS, TS, MT, TS, TT, KK, SA, and YN; Acquisition of data, TF, MK, HY, AH, KF, SK, JF, HM, YI, ST, TS, SY, TM, MA, HM, YA, HN, MO, HT, YA, IS, TS, MT, TS, TT, KK, SA, and YN; Supervision, MK, HM, HN, AT, and YN.; All authors approved the final version of the manuscript.

Funding: This work was partially supported by the Grants-in-Aid for Young Scientists (grant number JP18K16626) from the Japan Society for the Promotion of Science.

Institutional Review Board Statement: This study approval was obtained from the Kyushu University Institutional Review Board (approval number: 29-277). The study was conducted in accordance with the Declaration of Helsinki.

Conflict of interest: All authors declare that they have no conflicts of interest.

\section{References}

1. Smolen JS, Breedveld FC, Burmester GR, Bykerk V, Dougados M, Emery P, et al. Treating rheumatoid arthritis to target: 2014 update of the recommendations of an international task force. Ann Rheum Dis. 2016;75(1):3-15. 
2. Aletaha D, Smolen JS. Diagnosis and Management of Rheumatoid Arthritis: A Review. Jama. 2018;320(13):1360-72.

3. Barton JL, Imboden J, Graf J, Glidden D, Yelin EH, Schillinger D. Patient-physician discordance in assessments of global disease severity in rheumatoid arthritis. Arthritis Care Res (Hoboken). 2010;62(6):857-64.

4. Cho SK, Sung YK, Choi CB, Bang SY, Cha HS, Choe JY, et al. What factors affect discordance between physicians and patients in the global assessment of disease activity in rheumatoid arthritis? Mod Rheumatol. 2017;27(1):35-41.

5. Lindström Egholm C, Krogh NS, Pincus T, Dreyer L, Ellingsen T, Glintborg B, et al. Discordance of Global Assessments by Patient and Physician Is Higher in Female than in Male Patients Regardless of the Physician's Sex: Data on Patients with Rheumatoid Arthritis, Axial Spondyloarthritis, and Psoriatic Arthritis from the DANBIO Registry. J Rheumatol. 2015;42(10):1781-5.

6. Smolen JS, Strand V, Koenig AS, Szumski A, Kotak S, Jones TV. Discordance between patient and physician assessments of global disease activity in rheumatoid arthritis and association with work productivity. Arthritis Res Ther. 2016;18(1):114.

7. Kaneko Y, Sato M, Cai Z, Sato M. Assessment of discordance of treatment satisfaction between patients with rheumatoid arthritis in low disease activity or in remission and their treating physicians: A cross-sectional survey. Mod Rheumatol. 2021;31(2):326-33.

8. Bartlett SJ, De Leon E, Orbai AM, Haque UJ, Manno RL, Ruffing V, et al. Patient-reported outcomes in RA care improve patient communication, decision-making, satisfaction and confidence: qualitative results. Rheumatology (Oxford). 2020;59(7):1662-70.

9. Kelly A, Tymms K, Tunnicliffe DJ, Sumpton D, Perera C, Fallon K, et al. Patients' Attitudes and Experiences of Disease-Modifying Antirheumatic Drugs in Rheumatoid Arthritis and Spondyloarthritis: A Qualitative Synthesis. Arthritis Care Res (Hoboken). 2018;70(4):525-32.

10. Schäfer M, Albrecht K, Kekow J, Rockwitz K, Liebhaber A, Zink A, et al. Factors associated with treatment satisfaction in patients with rheumatoid arthritis: data from the biological register RABBIT. RMD Open. 2020;6(3).

11. Mahlich J, Schaede U, Sruamsiri R. Shared Decision-Making and Patient Satisfaction in Japanese Rheumatoid Arthritis Patients: A New "Preference Fit" Framework for Treatment Assessment. Rheumatol Ther. 2019;6(2):269-83.

12. Jiang N, Yang P, Liu S, Li H, Wu L, Shi X, et al. Satisfaction of Patients and Physicians with Treatments for Rheumatoid Arthritis: A Population-Based Survey in China. Patient Prefer Adherence. 2020;14:1037-47.

13. Taylor PC, Ancuta C, Nagy O, de la Vega MC, Gordeev A, Janková R, et al. Treatment Satisfaction, Patient Preferences, and the Impact of Suboptimal Disease Control in a Large International Rheumatoid Arthritis Cohort: SENSE Study. Patient Prefer Adherence. 2021;15:359-73.

14. Marshall NJ, Wilson G, Lapworth K, Kay LJ. Patients' perceptions of treatment with anti-TNF therapy for rheumatoid arthritis: a qualitative study. Rheumatology (Oxford). 2004;43(8):1034-8.

15. Wolfe F, Michaud K. Resistance of rheumatoid arthritis patients to changing therapy: discordance between disease activity and patients' treatment choices. Arthritis Rheum. 2007;56(7):2135-42.

16. Lofland JH, Johnson PT, Ingham MP, Rosemas SC, White JC, Ellis L. Shared decision-making for biologic treatment of autoimmune disease: influence on adherence, persistence, satisfaction, and health care costs. Patient Prefer Adherence. 2017;11:947-58. 
17. Li HB, Wu LJ, Jiang N, Yang PT, Liu SY, Shi XF, et al. Treatment satisfaction with rheumatoid arthritis in patients with different disease severity and financial burden: A subgroup analysis of a nationwide survey in China. Chin Med J (Engl). 2020;133(8):892-8.

18. Oyamada A, Matsumoto Y, Wakata Y, Kimura A, Ikuta K, Tsuchiya K, et al. Characteristics of patients with fragility hip fractures in the northern Kyushu district in Japan: a multicenter prospective registry based on an electronic data capture system. J Bone Miner Metab. 2018;36(5):596-604.

19. Haraguchi A, Nakashima Y, Miyahara H, Esaki Y, Okazaki K, Fukushi JI, et al. Minimum 10-year results of cementless total hip arthroplasty in patients with rheumatoid arthritis. Mod Rheumatol. 2017;27(4):598-604.

20. Fujiwara T, Fujimura K, Hamai S, Kamura S, Nakashima Y, Miyahara H. Mid-term clinical outcome of constrained condylar knee prosthesis for patients with rheumatoid arthritis. Mod Rheumatol. 2019;29(4):596601.

21. Pincus T, Summey JA, Soraci SA, Jr., Wallston KA, Hummon NP. Assessment of patient satisfaction in activities of daily living using a modified Stanford Health Assessment Questionnaire. Arthritis Rheum. 1983;26(11):134653.

22. EuroQol-a new facility for the measurement of health-related quality of life. Health Policy. 1990;16(3):199-208.

23. Hamashima C, Yoshida K. A study of the reliability of health state valuations in the Japanese EuroQol instrument. Environ Health Prev Med. 2001;6(3):189-91.

24. Hosmer Jr DW LS, Sturdivant RX. Applied Logistic Regression, Third Edition: Wiley Series in Probability and Statistics; 2013.

25. Tatangelo M, Tomlinson G, Paterson JM, Keystone E, Bansback N, Bombardier C. Health care costs of rheumatoid arthritis: A longitudinal population study. PLoS One. 2021;16(5):e0251334.

26. Hoshi D, Tanaka E, Igarashi A, Inoue E, Kobayashi A, Sugimoto N, et al. Profiles of EQ-5D utility scores in the daily practice of Japanese patients with rheumatoid arthritis; Analysis of the IORRA database. Mod Rheumatol. 2016;26(1):40-5.

27. van der Heijde D, Klareskog L, Singh A, Tornero J, Melo-Gomes J, Codreanu C, et al. Patient reported outcomes in a trial of combination therapy with etanercept and methotrexate for rheumatoid arthritis: the TEMPO trial. Ann Rheum Dis. 2006;65(3):328-34.

28. Matsuura E, Origuchi T, Hamasaki M. Association of health-related quality of life with self-management and satisfaction of relationship with healthcare professionals in female patients with rheumatoid arthritis. Mod Rheumatol. 2021:1-18.

29. Chen MH, Lee MH, Liao HT, Chen WS, Lai CC, Tsai CY. Health-related quality of life outcomes in patients with rheumatoid arthritis and ankylosing spondylitis after tapering biologic treatment. Clin Rheumatol. 2018;37(2):429-38.

30. Nakashima Y, Kondo M, Shono E, Ishinishi T, Tsukamoto H, Kuroda K, et al. Suppression of joint destruction with subcutaneous tocilizumab for Japanese patients with rheumatoid arthritis in clinical practice. Mod Rheumatol. 2020;30(5):807-15.

31. Smolen JS, Landewé RBM, Bijlsma JWJ, Burmester GR, Dougados M, Kerschbaumer A, et al. EULAR recommendations for the management of rheumatoid arthritis with synthetic and biological disease-modifying antirheumatic drugs: 2019 update. Ann Rheum Dis. 2020;79(6):685-99.

32. Jørgensen KK, Olsen IC, Goll GL, Lorentzen M, Bolstad N, Haavardsholm EA, et al. Switching from originator infliximab to biosimilar CT-P13 compared with maintained treatment with originator infliximab (NOR-SWITCH): 
a 52-week, randomised, double-blind, non-inferiority trial. Lancet. 2017;389(10086):2304-16.

33. Smolen JS, Choe JY, Prodanovic N, Niebrzydowski J, Staykov I, Dokoupilova E, et al. Safety, immunogenicity and efficacy after switching from reference infliximab to biosimilar SB2 compared with continuing reference infliximab and SB2 in patients with rheumatoid arthritis: results of a randomised, double-blind, phase III transition study. Ann Rheum Dis. 2018;77(2):234-40.

34. Yoo DH, Prodanovic N, Jaworski J, Miranda P, Ramiterre E, Lanzon A, et al. Efficacy and safety of CT-P13 (biosimilar infliximab) in patients with rheumatoid arthritis: comparison between switching from reference infliximab to CT-P13 and continuing CT-P13 in the PLANETRA extension study. Ann Rheum Dis. 2017;76(2):355-63.

35. Glintborg B, Loft AG, Omerovic E, Hendricks O, Linauskas A, Espesen J, et al. To switch or not to switch: results of a nationwide guideline of mandatory switching from originator to biosimilar etanercept. One-year treatment outcomes in 2061 patients with inflammatory arthritis from the DANBIO registry. Ann Rheum Dis. 2019;78(2):192-200.

36. Glintborg B, Sørensen IJ, Loft AG, Lindegaard H, Linauskas A, Hendricks O, et al. A nationwide non-medical switch from originator infliximab to biosimilar CT-P13 in 802 patients with inflammatory arthritis: 1-year clinical outcomes from the DANBIO registry. Ann Rheum Dis. 2017;76(8):1426-31.

37. Smolen JS, Caporali R, Doerner T, Fautrel B, Benedetti F, Pieper B, et al. Treatment journey in rheumatoid arthritis with biosimilars: from better access to good disease control through cost savings and prevention of nocebo effects. RMD Open. 2021;7(2).

38. Radawski C, Genovese MC, Hauber B, Nowell WB, Hollis K, Gaich CL, et al. Patient Perceptions of Unmet Medical Need in Rheumatoid Arthritis: A Cross-Sectional Survey in the USA. Rheumatol Ther. 2019;6(3):461-71.

39. Maassen JM, Dos Santos Sobrín R, Bergstra SA, Goekoop R, Huizinga TWJ, Allaart CF. Glucocorticoid discontinuation in patients with early rheumatoid and undifferentiated arthritis: a post-hoc analysis of the BeSt and IMPROVED studies. Ann Rheum Dis. 2021;80(9):1124-9.

40. Chester Wasko M, Dasgupta A, Ilse Sears G, Fries JF, Ward MM. Prednisone Use and Risk of Mortality in Patients With Rheumatoid Arthritis: Moderation by Use of Disease-Modifying Antirheumatic Drugs. Arthritis Care Res (Hoboken). 2016;68(5):706-10.

41. Schneeweiss S, Setoguchi S, Weinblatt ME, Katz JN, Avorn J, Sax PE, et al. Anti-tumor necrosis factor alpha therapy and the risk of serious bacterial infections in elderly patients with rheumatoid arthritis. Arthritis Rheum. 2007;56(6):1754-64.

42. Fujiwara T, Tokuda K, Momii K, Shiomoto K, Tsushima H, Akasaki Y, et al. Prognostic factors for the short-term mortality of patients with rheumatoid arthritis admitted to intensive care units. BMC Rheumatol. 2020;4(1):64.

43. Wilson JC, Sarsour K, Gale S, Pethö-Schramm A, Jick SS, Meier CR. Incidence and Risk of GlucocorticoidAssociated Adverse Effects in Patients With Rheumatoid Arthritis. Arthritis Care Res (Hoboken). 2019;71(4):498-511.

44. Roodenrijs NMT, de Hair MJH, van der Goes MC, Jacobs JWG, Welsing PMJ, van der Heijde D, et al. Characteristics of difficult-to-treat rheumatoid arthritis: results of an international survey. Ann Rheum Dis. 2018;77(12):1705-9.

45. Hickson M, Frost G. An investigation into the relationships between quality of life, nutritional status and physical function. Clin Nutr. 2004;23(2):213-21. 
46. Boccaccio DE, Cenzer I, Covinsky KE. Life satisfaction among older adults with impairment in activities of daily living. Age Ageing. 2021.

47. Aletaha D, Ward MM. Duration of rheumatoid arthritis influences the degree of functional improvement in clinical trials. Ann Rheum Dis. 2006;65(2):227-33.

\section{Figures}

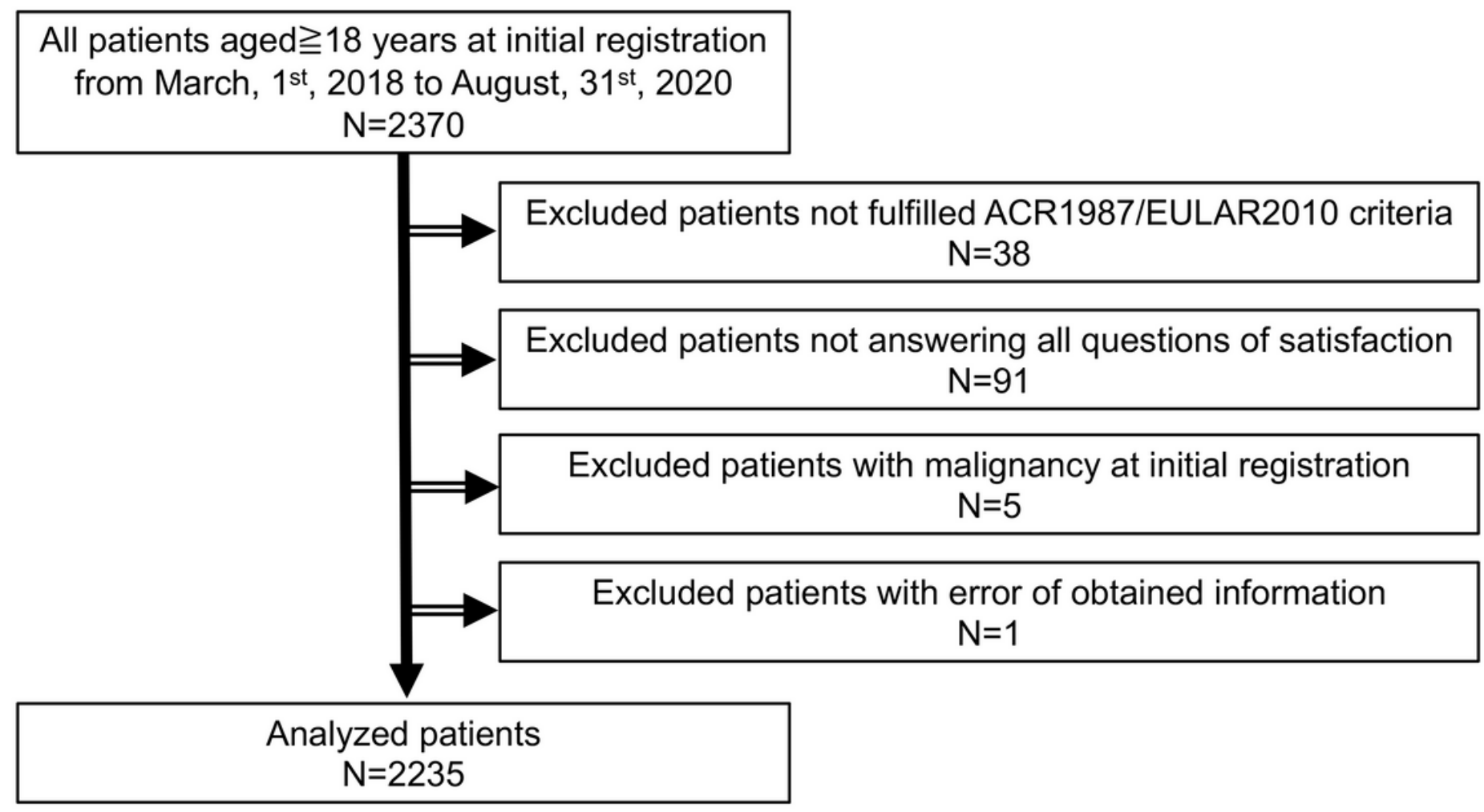

Figure 1

Flowchart of the selection of rheumatoid arthritis patients from the initial registration data of the FRANK registry. 

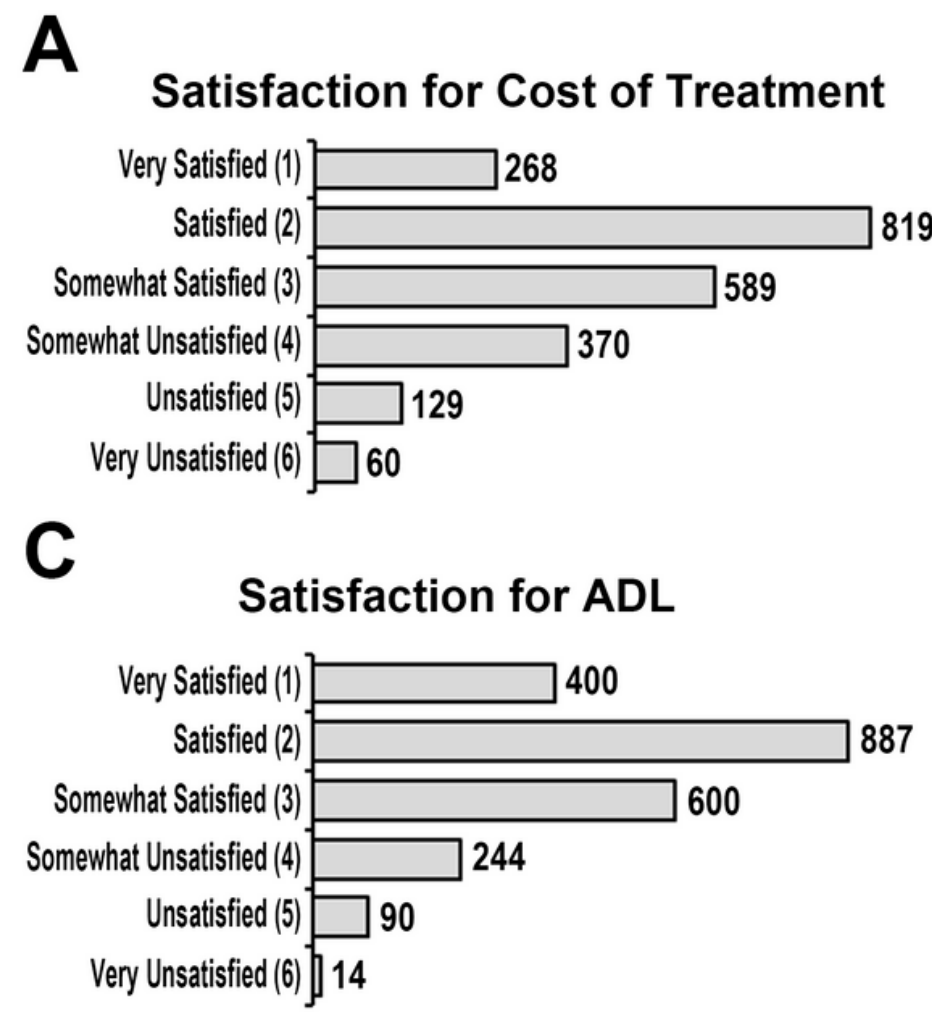

B

\section{Satisfaction for Effect of Treatment}

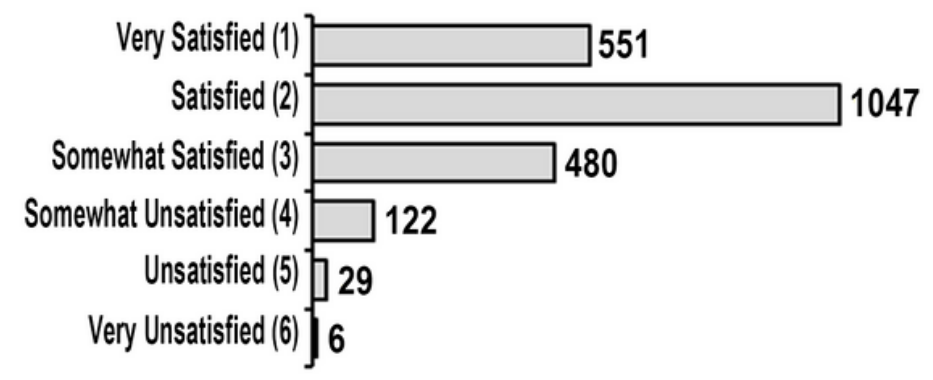

D

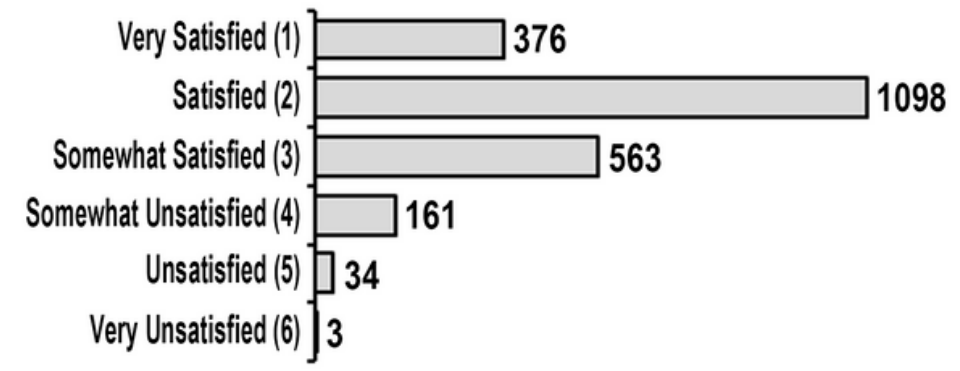

Figure 2

Distribution of satisfaction ratings from the initial registration data of FRANK registry. Each self-administered satisfaction rating was evaluated via a 6-step score (very satisfied: 1 point; satisfied: 2 points; somewhat satisfied: 3 points; somewhat unsatisfied: 4 points; unsatisfied: 5 points; very unsatisfied: 6 points). (A) Satisfaction for cost of treatment, (B) satisfaction for effect of treatment, (C) satisfaction for ADL, and (D) satisfaction for global treatment. 